\title{
Global Dynamic Analysis of a Vector-Borne Plant Disease Model with Discontinuous Treatment
}

\author{
Hengmin Lv $^{1 *}$, Lizhi Fei ${ }^{1,2}$, Zhen Yuan1, Fumin Zhang ${ }^{3}$ \\ ${ }^{1}$ Department of Basic Course Education, Ji'an College, Ji'an, China \\ ${ }^{2}$ College of Mathematics, Sichuan University, Chengdu, China \\ ${ }^{3}$ Key Laboratory of Jiangxi Province for Numerical Simulation and Emulation Techniques, Gannan Normal University, Ganzhou, \\ China \\ Email: *lvhengmin2005@163.com
}

How to cite this paper: Lv, H.M., Fei, L.Z., Yuan, Z. and Zhang, F.M. (2018) Global Dynamic Analysis of a Vector-Borne Plant Disease Model with Discontinuous Treatment. Applied Mathematics, 9, 496-511. https://doi.org/10.4236/am.2018.95036

Received: May 2, 2018

Accepted: May 25, 2018

Published: May 28, 2018

Copyright (C) 2018 by authors and Scientific Research Publishing Inc. This work is licensed under the Creative Commons Attribution International License (CC BY 4.0).

http://creativecommons.org/licenses/by/4.0/

\section{Open Access}

\begin{abstract}
This paper proposes a vector-borne plant disease model with discontinuous treatment strategies. Constructing Lyapunov function and applying non-smooth theory to analyze discontinuous differential equations, the basic reproductive number $R_{0}$ is proved, which determines whether the plant disease will be extinct or not. If $R_{0}<1$, the existence and global stability of disease-free equilibrium is established; If $R_{0}>1$, there exists a unique endemic equilibrium which is globally stable. The numerical simulations are provided to verify our theoretical results, which indicate that after infective individuals reach some level, strengthening treatment measures is proved to be beneficial in controlling disease transmission.
\end{abstract}

\section{Keywords}

Vector-Borne, Plant Disease Model, Basic Reproduction Number, Discontinuous Treatment

\section{Introduction}

The plants play an important role in our lives, as most of our daily food, clothing and building materials come from plants. With the change of environment, there are outbreaks of plant diseases, which seriously affect the health of plants and people's life, such as huanglongbing [1], Blackleg [2]. We know that plant diseases have been responsible for the death and suffering of millions of people and countless animals [3]. Controlling the outbreak and spread of plant diseases has 
become the common goal of scientists. Available control measures include biological, cultural, and chemical methods [4]. Chemical control is a quite effective method, but the residues of chemical drugs have a direct negative impact on environment and thus are not encouraged. To effectively control plant disease and to reduce the harm to environment, it is crucial to understand disease transmission dynamics.

The prevention and control of plant infectious diseases is of vital importance in agricultural production [5]. To work with the plant disease, we first understand how they spread. There are many ways that plant viruses interact with the vectors; this transmission works in the following way. The vectors consume sap from an infected host through their stylet. When the infected vector contacts a healthy plant, some virus particles leave the vector and invade the plant [6]. So the vector-borne is a very important part of the transmission of plant diseases.

Treatment plays a very important role to control the spread of diseases. In recent years, many researchers [7] [8] [9] [10] have studied some mathematical models incorporating treatment. For example, in [11], Wang and Ruan studied an epidemic model, and provide the limited resources for the treatment of patients. In [12], Wang proposed constant treatment, which simulates a limited capacity for treatment. In practice, when the number of infectives is large, the constant treatment is suitable for hypothesis of model. Recently, discontinuous treatment strategies are proposed by [13] [14]. The results show that discontinuous treatment strategies would be accord with real condition. Applying this discontinuous treatment strategy makes the mathematical model a discontinuous system. At the same time, some non-smooth analysis techniques [15] are used for this system.

In [16], Shi and Zhao presented a vector-borne plant disease model, but they do not studied treatment to the infected plant host. Treating infected plant is a quite effective method which to control the outbreak of the plant disease. Although continuous treatment is an effective method, the outbreak of the plant disease is periodic, and continuing treatment can be a huge waste of resources. In order to be realistic, we built a vector-borne plant disease model with discontinuous treatment.

The paper is organized as follows. In the next section, we will construct the model and introduce the rational assumptions for model. In Section 3, positivity of the solution for the model will be clearly discussed. We obtain the existence of possible equilibria, the basic reproductive number, and the stability of equilibria in Section 4. In Section 5 and Section 6, we summarize our main results and main results are numerically simulated.

\section{Model and Preliminaries}

To construct the model, the following assumptions are being made by Shi et al. in [16]. 
(A1) The total of the insect vector population is divided into $X$ and $Y$, which denotes the densities of the susceptible vector and infective vector at time $t$, respectively. The total of the plant host population is divided into $S, I$, and $R$, which represents the numbers of the susceptible, infective, and recovered host plant population at time $t$, respectively. At the same time, we assume that the number of plants in one area is fixed. The total number of plants $K=S+I+R$ is a positive constant. In fact, when a plant has died, it would be replaced by a new plant to keep the total number of plants. Further, we assume that those new plants are susceptible, i.e., we chose the birth rate of susceptible plant host as $f(S, I)=\mu K+d I$.

(A2) The susceptible plants can be infected not only by the infected insect vectors but also by the infected plants.

(A3) A susceptible vector can be infected only by an infected plant host, and after it is infected, it will hold the virus for the rest of its life. Further, there is no vertical infection being considered.

(A4) The replenishment rate of insect vectors is a positive constant, and all of the new born vectors are susceptible.

According to the principle of the compartmental model, consider the following model with discontinuous treatment:

$$
\left\{\begin{array}{l}
\dot{S}=f(S, I)-\mu S-\left(\beta_{P} Y+\beta_{s} I\right) S, \\
\dot{I}=\left(\beta_{P} Y+\beta_{s} I\right) S-(d+\mu+\gamma) I-h(I), \\
\dot{R}=\gamma I+h(I)-\mu R, \\
\dot{X}=\Lambda-\beta_{1} I X-m X, \\
\dot{Y}=\beta_{1} I X-m Y .
\end{array}\right.
$$

Here the dimensionless variables and parameters (with parameter values) are given in Table 1 .

The function $h(I)=\varphi(I) I$ represents the treatment rate. $\varphi(I)$ satisfies the following assumptions. Obviously, the treatment rate should be nondecreasing as the number of infectious individuals is increasing. The following assumption will be needed throughout the paper.

$\left(\mathrm{H}_{1}\right) \quad \varphi:[0, \infty) \rightarrow[0, \infty)$ is nondecreasing and has at most a finite number of jump discontinuities in every compact interval. No loss of generality, we always assume that $\varphi$ is continuous at $I=0$, otherwise we define $\varphi(0)$ to be $\varphi\left(0^{+}\right)$. Here $\varphi\left(0^{+}\right)$denotes the right limit of $\varphi(I)$ as $I \rightarrow 0^{+}$.

By adding the fourth and fifth equations of system (2.1), we get

$$
\dot{N}=\Lambda-m N
$$

where $N=X+Y$. From Equation (2.2), we easily get $N \rightarrow \frac{\Lambda}{m}$ as $t \rightarrow \infty$.

Note that $S+I+R=K$. Since the variable $R$ and $X$ does not appear in the first two equations of model (2.1), meanwhile, let's substitute $X$ for $\left(\frac{\Lambda}{m}-Y\right)$ in the fifth equation. We only need to study the first two equations and the fifth 
Table 1. Dimensionless variables and parameters (with illustrative parameter values) in system (2.1).

\begin{tabular}{ccc}
\hline Parameter & Description & Default value \\
\hline$S$ & number of the susceptible plant hosts & - \\
$I$ & number of the infected plant hosts & - \\
$K$ & number of the recovered plant hosts & - \\
$K$ & sum of the total plant hosts & $50-1000$ \\
$Y$ & density of the susceptible insect vectors &.. \\
$N$ & density of the infected insect vectors & - \\
$\beta_{1}$ & sum of the total insect vectors density & $50-100$ \\
$\beta_{P}$ & biting rate of an infected vector on the susceptible host plants & $0.01-0.02$ \\
$\beta_{S}$ & infection incidence between infected and susceptible hosts & $0.01-0.02$ \\
$\gamma$ & the conversion rate of infected hosts to recovered hosts & $0-0.4$ \\
$\mu$ & natural death rate of plant hosts & $0-0.1$ \\
$\Lambda$ & birth or immigration of insect vectors & 5 \\
$m$ & natural death rate of insect vectors & $0-0.5$ \\
$d$ & disease-induced mortality of infected hosts & 0.1 \\
\hline
\end{tabular}

equation of model (2.1), thereby lowering the order of the system to be studied, i.e.

$$
\left\{\begin{array}{l}
\dot{S}=\mu(K-S)-\left(\beta_{P} Y+\beta_{s} I\right) S+d I, \\
\dot{I}=\left(\beta_{P} Y+\beta_{s} I\right) S-\omega I-h(I), \\
\dot{Y}=\frac{\Lambda \beta_{1} I}{m}-\left(\beta_{1} I+m\right) Y .
\end{array}\right.
$$

where $\omega=d+\mu+\gamma$. Obviously,

$$
\Omega=\left\{(S, I, Y) \in R_{+}^{3}: 0 \leq S+I \leq K, 0 \leq Y \leq \frac{\Lambda}{m}\right\}
$$

is the positively invariant set for system (2.3).

According to the definition of solutions for differential equations with discontinuous right-hand sides in [15] [17], $(S(t), I(t), Y(t))$ is called a solution with initial condition

$$
(S(0), I(0), Y(0))=\left(S_{0}, I_{0}, Y_{0}\right), S_{0}, I_{0}, Y_{0} \geq 0
$$

of model (2.3) on $[0, T), 0<T \leq \infty$, if it is absolutely continuous on any compact subinterval of $[0, T)$, and almost everywhere on $[0, T)$ (abbreviated to a.e. on $[0, T))$ satisfies the following differential inclusion:

$$
\left\{\begin{array}{l}
\dot{S}=\mu(K-S)-\left(\beta_{P} Y+\beta_{s} I\right) S+d I, \\
\dot{I} \in\left(\beta_{P} Y+\beta_{s} I\right) S-\omega I-\overline{c o}[h(I)], \\
\dot{Y}=\frac{\Lambda \beta_{1} I}{m}-\left(\beta_{1} I+m\right) Y .
\end{array}\right.
$$


where $\overline{c o}[h(I)]=[h(I-0), h(I+0)]$. Here, $h(I-0)$ and $h(I+0)$ denote the left limit and the right limit of the function $h(I)$ at $I$, respectively.

From $\left(\mathrm{H}_{1}\right)$, it is clear that the set map

$$
\begin{aligned}
& (S, I, Y) \mapsto\left(\mu(K-S)-\left(\beta_{P} Y+\beta_{s} I\right) S+d I,\right. \\
& \left.\left(\beta_{P} Y+\beta_{s} I\right) S-\omega I-\overline{c o}[h(I)], \frac{\Lambda \beta_{1} I}{m}-\left(\beta_{1} I+m\right) Y\right)
\end{aligned}
$$

is an upper semi-continuous set-valued map with non-empty compact convex values. By the measurable selection theorem [15], if $(S(t), I(t), Y(t))$ is a solution of model (2.3) on $[0, T)$, then there is a measurable function $m(t) \in \overline{c o}[h(I(t))]$ such that

$$
\left\{\begin{array}{l}
\dot{S}=\mu(K-S)-\left(\beta_{P} Y+\beta_{s} I\right) S+d I \\
\dot{I} \in\left(\beta_{P} Y+\beta_{s} I\right) S-\omega I-m(t), \text { a.e. on }[0, T) . \\
\dot{Y}=\frac{\Lambda \beta_{1} I}{m}-\left(\beta_{1} I+m\right) Y .
\end{array}\right.
$$

\section{Positivity}

In this section, we will prove the positive of the solution to the initial condition of the model (2.3) with positive initial value. First, we will prove the following theorem.

Theorem 3.1. Suppose that assumption $\left(\mathrm{H}_{1}\right)$ holds and let $(S(t), I(t), Y(t))$ be the solution with initial condition (2.5) of model (2.3) on $[0, T)$. Then $(S(t), I(t), Y(t))$ is nonnegative on $[0, T)$.

Proof: By the definition of a solution of (2.3) in the sense of Filippov, $(S(t), I(t), Y(t))$ must be a solution to differential inclusion (2.6). From the first equation of (2.6), we have

$$
\begin{aligned}
& {\left[S_{0}+\int_{0}^{t}(\mu k+d I(u)) \exp \left(\int_{0}^{u}\left(\mu+\beta_{P} Y(\rho)+\beta_{s} I(\rho)\right) \mathrm{d} \rho\right) \mathrm{d} u\right]} \\
& \cdot \exp \left(-\left(\int_{0}^{t}\left(\mu+\beta_{P} Y(\rho)+\beta_{s} I(\rho)\right) \mathrm{d} \rho\right)\right)>0
\end{aligned}
$$

for all $t \in(0, T)$.

Based on the previous hypothesis of $\left(\mathrm{H}_{1}\right)$, we have $\overline{c o}[h(0)]=0$ and $h(I)$ is continuous at $I=0$. Combining the continuity of $\varphi$ at $I=0$, it may be concluded that there exists a positive constant $\delta$ such that $\varphi(I)$ is continuous as $|I|<\delta$. On this account, when $|I|<\delta$ the differential inclusion (2.6) becomes the following system of differential equations:

$$
\left\{\begin{array}{l}
\dot{I}=\left(\beta_{P} Y+\beta_{s} I\right) S-(\omega+\varphi(I)) I, \\
\dot{Y}=\frac{\Lambda \beta_{1} I}{m}-\left(\beta_{1} I+m\right) Y .
\end{array}\right.
$$

We divide this into four cases to discuss the positivity of the solutions for (2.6).

1) $I_{0}=Y_{0}=0$. 
From (3.2), we see that $I(t)=Y(t)=0$ for all $t \in[0, T)$.

2) $I_{0}>0, Y_{0}=0$.

By the continuity of $I(t)$ at $t=0$ and $\left.\frac{\mathrm{d} Y}{\mathrm{~d} t}\right|_{t=0}=\frac{\Lambda \beta_{1} I_{0}}{m}>0$, we conclude $I(t)>0$ and $Y(t)>0$ for all $t \in(0, T)$. If it is not true, then we can set

$$
t_{1}=\inf \{t: I(t)=0 \text { or } Y(t)=0\} \in(0, T) .
$$

If $I\left(t_{1}\right)=0$, then from $\frac{\mathrm{d} I}{\mathrm{~d} t} \geq-(\omega+\varphi(I)) I$ for $0 \leq t \leq t_{1}$, we have $I\left(t_{1}\right) \geq I_{0} \exp \left(-(\omega+\varphi(I)) t_{1}\right)>0$. This is a contradiction.

If $I\left(t_{1}\right)=0$, then there is a $\theta$ such that $t_{1}-\theta>0$ and $0<I(t)<\delta$ on $\left[t-\theta, t_{1}\right)$. Therefore, the second equation of (3.2) implies

$$
\frac{\mathrm{d} Y}{\mathrm{~d} t} \geq-\left(\beta_{1} I+m\right) Y
$$

We have

$$
Y\left(t_{1}\right) \geq Y\left(t_{1}-\theta\right) \exp \left(-\int_{t_{1}-\theta}^{t_{1}}\left(\beta_{1} I(\xi)+m\right) \mathrm{d} \xi\right)>0
$$

This is also a contradiction. Hence, $I(t)$ and $Y(t)$ are positive for all $t \in(0, T)$. The same conclusion can be reached for the following two cases.

3) $I_{0}=0, Y_{0}>0$.

4) $I_{0}>0, Y_{0}>0$. This completes the proof.

\section{The Equilibria and Their Stability}

In this section, we will discuss the existence of equilibria of system (2.3). First, we prove the existence of endemic equilibrium.

Let $(S(t), I(t), Y(t))=\left(S^{*}, I^{*}, Y^{*}\right)$ is a constant solution of (2.3), where $\left(S^{*}, I^{*}, Y^{*}\right)$ satisfies the following system:

$$
\left\{\begin{array}{l}
0=\mu\left(K-S^{*}\right)-\left(\beta_{P} Y^{*}+\beta_{s} I^{*}\right) S^{*}+d I^{*} \\
0 \in\left(\beta_{P} Y^{*}+\beta_{s} I^{*}\right) S^{*}-\omega I^{*}-\overline{c o}\left[h\left(I^{*}\right)\right], \\
0=\frac{\beta_{1} I^{*} \Lambda}{m}-\left(\beta_{1} I^{*}+m\right) Y^{*}
\end{array}\right.
$$

Since $h(0)=0$, there always exists a disease-free equilibrium $P_{0}$ of the model (2.3), where $P_{0}=(K, 0,0)$. Next, we consider that the existence of an endemic equilibrium of the model (2.3).

It follows from the first and third equations of (4.1), we conclude that

$$
S^{*}=\frac{d I^{*}+\mu K}{\mu+\beta_{P} Y^{*}+\beta_{s} I^{*}}, Y^{*}=\frac{\Lambda \beta_{1} I^{*}}{m\left(\beta_{1} I^{*}+m\right)} .
$$

Substituting (4.2) into the second inclusion of (4.1), we have the follows

$$
\frac{A_{1} I^{* 2}+B_{1} I^{*}+C_{1}}{A_{2} I^{* 2}+B_{2} I^{*}+C_{2}}-\omega \in \overline{c o}[\varphi(I)]=\left[\varphi\left(I^{*}-0\right), \varphi\left(I^{*}+0\right)\right]
$$


where

$$
\begin{gathered}
A_{1}=m d \beta_{1} \beta_{s} \\
B_{1}=d \Lambda \beta_{p} \beta_{1}+m^{2} d \beta_{s}+\mu m K \beta_{1} \beta_{s}, \\
C_{1}=\mu K\left(\Lambda \beta_{p} \beta_{1}+m^{2} \beta_{s}\right), \\
A_{2}=m \beta_{1} \beta_{s}, \\
B_{2}=\mu m \beta_{1}+\Lambda \beta_{1} \beta_{p}+m^{2} \beta_{s}, \\
C_{2}=\mu m^{2} .
\end{gathered}
$$

Denote

$$
g\left(I^{*}\right)=\frac{A_{1} I^{* 2}+B_{1} I^{*}+C_{1}}{A_{2} I^{* 2}+B_{2} I^{*}+C_{2}}-\omega
$$

and let

$$
R_{0}=\frac{K\left(\Lambda \beta_{p} \beta_{1}+\beta_{s} m^{2}\right)}{m^{2}(\omega+\varphi(0))}
$$

We next claim that $R_{0}$ is the basic reproductive number for the model (2.3) which will determine the existence of an endemic equilibrium.

Theorem 4.1. Suppose that assumption (H1) holds. If $R_{0} \leq 1$, then there only exists a disease-free equilibrium $P_{0}(K, 0,0)$. If $R_{0}>1$, then there exists a unique positive endemic equilibrium $P^{*}\left(S^{*}, E^{*}, I^{*}\right)$ except $P_{0}$.

Proof: By $R_{0} \leq 1$, we get $g(0) \leq \varphi(0)$. Since $g(I)$ is nonincreasing on $I$ and $\varphi(I)$ is nondecreasing on $I$. For this reason, the inclusion (4.3) is only valid at $I=0$. Hence, the model (2.3) has a unique disease-free equilibrium as long as $R_{0} \leq 1$.

From (4.4), we have the following

$$
A I^{2}+B I+C=0
$$

where

$$
\begin{gathered}
A=m \beta_{1} \beta_{s}(m d-\omega)<0, \\
B=\Lambda d \beta_{1} \beta_{p}+m^{2} d \beta_{s}+\mu m K \beta_{1} \beta_{s}-\omega m \mu \beta_{1}-\Lambda \omega \beta_{1} \beta_{p}-\omega m^{2} \beta_{s}, \\
C=\mu \omega m^{2}\left(R_{0}-1\right)+\mu m^{2} \varphi(0) .
\end{gathered}
$$

If $R_{0} \geq 1$, then $C>0$, and the Equation (4.6) has a unique positive root $I$, where

$$
I=\frac{-B+\Delta^{\frac{1}{2}}}{2 A}, \Delta=B^{2}-4 A C .
$$

If $R_{0}>1$, then $g(0)>\varphi(0) \geq 0$. Meanwhile, the inequality $I \geq I_{1}$, it implies $g(I) \leq 0$. Therefore, the set

$$
\Gamma=\{I: g(I) \geq \varphi(I+0), I>0\}
$$

is bounded and non-empty. We can write 


$$
I^{*}=\sup \{I: g(I) \geq \varphi(I+0), I>0\}
$$

It follows easily that

$$
g\left(I^{*}\right) \geq \varphi\left(I^{*}+0\right) \text { and } 0<I^{*} \leq I_{0} .
$$

We claim $g\left(I^{*}\right) \in\left[\varphi\left(I^{*}-0\right), \varphi\left(I^{*}+0\right)\right]$. Assumption, contrary to our claim, that

$$
g\left(I^{*}\right)>\varphi\left(I^{*}+0\right)=\lim _{I \rightarrow I^{*}+0} \varphi(I)
$$

From $\left(\mathrm{H}_{1}\right)$, there exists a $\delta>0$ such that

$$
g\left(I^{*}+\delta\right)>\varphi\left(I^{*}+\delta\right)=\varphi\left(I^{*}+\delta+0\right) .
$$

This contradicts the definition of $I^{*}$. Thus, we have $g\left(I^{*}\right) \in\left[\varphi\left(I^{*}-0\right), \varphi\left(I^{*}+0\right)\right]$. That is to say, $I^{*}$ is a positive solution of the inclusion (4.3). We proceed to show that $I^{*}$ is the only one positive solution of the inclusion (4.3). If the inclusion (4.3) has another positive solution $I^{* * *}$, then there must exist two numbers

$$
\eta^{*} \in\left[\varphi\left(I^{*}\right)\right] \text { and } \eta^{* *} \in\left[\varphi\left(I^{* *}\right)\right]
$$

which satisfy

$$
\frac{A_{1} I^{* 2}+B_{1} I^{*}+C_{1}}{A_{2} I^{* 2}+B_{2} I^{*}+C_{2}}-\omega=\eta^{*}
$$

and

$$
\frac{A_{1} I^{* * 2}+B_{1} I^{*}+C_{1}}{A_{2} I^{* * 2}+B_{2} I^{* *}+C_{2}}-\omega=\eta^{* *}
$$

Subtracting (4.13) from (4.12) gives

$$
\begin{aligned}
& \frac{\left(A_{1} B_{2}-A_{2} B_{1}\right)\left(I^{*}-I^{* *}\right) I^{*} I^{* *}+\left(A_{1} C_{2}-A_{2} C_{1}\right)\left(I^{* 2}-I^{* * 2}\right)+\left(B_{1} C_{2}-B_{2} C_{1}\right)\left(I^{*}-I^{* *}\right)}{\left(A_{2} I^{* 2}+B_{2} I^{*}+C_{2}\right)\left(A_{2} I^{* * 2}+B_{2} I^{* *}+C_{2}\right)} \\
& =\eta^{*}-\eta^{* *}
\end{aligned}
$$

which implies

$$
\frac{\eta^{*}-\eta^{* *}}{I^{*}-I^{* *}}<0
$$

This is a contradiction. Hence, $I^{*}$ is the unique positive solution of the inclusion (4.3). Combining it with (4.2), we conclude that the $\left(S^{*}, I^{*}, Y^{*}\right)$ is the unique endemic equilibrium of (2.3). The proof is completed.

Next, we prove the global stability of the disease-free equilibrium and the endemic equilibrium. We do this in several steps. We first investigate the local properties of the equilibria of system (2.3).

Theorem 4.2. Assume $\left(\mathrm{H}_{1}\right)$ holds. The disease-free equilibrium $P_{0}$ is locally asymptotically stable if $R_{0}<1$, and is unstable if $R_{0}>1$.

Proof: We analyze the stability of the disease-free equilibrium by investigating 
the eigenvalues of the Jacobian matrix of model (2.3) at $P_{0}$. The matrix is

$$
J\left(P_{0}\right)=\left(\begin{array}{ccc}
-\mu & -\left(\beta_{s} K-d\right) & -\beta_{p} K \\
0 & \beta_{s} K-\omega-\varphi(0) & \beta_{p} K \\
0 & \frac{\Lambda \beta_{1}}{m} & -m
\end{array}\right)
$$

Thus, the characteristic equation at the disease-free equilibrium $P_{0}$ is

$$
J\left(P_{0}\right)=\left|\begin{array}{ccc}
-\mu-\lambda & -\left(\beta_{s} K-d\right) & -\beta_{p} K \\
0 & \beta_{s} K-\omega-\varphi(0)-\lambda & \beta_{p} K \\
0 & \frac{\Lambda \beta_{1}}{m} & -m-\lambda
\end{array}\right|
$$

It is easy to see that one of the roots with respect to $\lambda$ of (4.14) is $\lambda_{1}=-\mu<0$. the other two roots are determined by the following characteristic equation of $J\left(P_{0}\right)$ :

$$
\lambda^{2}+\lambda\left(\omega+\varphi(0)-\beta_{s} K\right)+m\left(\omega+\varphi(0)-\beta_{s} K\right)-\frac{\Lambda K \beta_{p} \beta_{1}}{m}=0 .
$$

From (4.18) and Routh-Hurwitz criteria [18], it is easily seen that both the real parts of $\lambda_{2}$ and of $\lambda_{3}$ are negative when $R_{0}<1$. When $R_{0}>1$, one of $\lambda_{2}$ and $\lambda_{3}$ is a number with a positive real part. Thus the disease-free equilibrium is locally asymptotically stable if $R_{0}<1$ and unstable if $R_{0}>1$.

We have shown that there exists a positive endemic equilibrium if and only if $R_{0}>1$ in Theorem 4.1. Here, we will establish its local stability.

Theorem 4.3. Suppose that assumption (H1) holds. If $R_{0}>1$, the endemic equilibrium $P^{*}$ of the system (2.3) is locally asymptotically stable.

Proof: The Jacobian matrix of (2.3) at the endemic equilibrium $P^{*}=\left(S^{*}, I^{*}, Y^{*}\right)$ is

$$
J\left(P^{*}\right)=\left(\begin{array}{ccc}
-\mu-\beta_{p} Y^{*}-\beta_{s} I^{*} & -\left(\beta_{s} S^{*} K-d\right) & -\beta_{p} S^{*} \\
\beta_{p} Y^{*}+\beta_{s} I^{*} & \beta_{s} S^{*}-\omega-\varphi^{\prime}\left(I^{*}\right) I^{*}-\varphi\left(I^{*}\right) & \beta_{p} S^{*} \\
0 & \frac{\Lambda \beta_{1}}{m}-\beta_{1} Y^{*} & -m
\end{array}\right)
$$

Replacing $-\mu-\beta_{p} Y^{*}-\beta_{s} I^{*}, \quad \beta_{s} S^{*}-\omega-\varphi\left(I^{*}\right)$ by $\mu K+d I^{*}, \quad \beta_{p} S^{*} Y^{*} / I^{*}$, respectively. So we have

$$
J\left(P^{*}\right)=\left(\begin{array}{ccc}
\mu K+d I^{*} & -\left(\beta_{s} S^{*} K-d\right) & -\beta_{p} S^{*} \\
\beta_{p} Y^{*}+\beta_{s} I^{*} & -\frac{\beta_{p} S^{*} Y^{*}}{I^{*}}-\varphi^{\prime}\left(I^{*}\right) I^{*} & \beta_{p} S^{*} \\
0 & \frac{\Lambda \beta_{1}}{m}-\beta_{1} Y^{*} & -m
\end{array}\right)
$$

The characteristic equation of $J\left(P^{*}\right)$ is 


$$
\lambda^{3}+a_{1} \lambda^{2}+a_{2} \lambda+a_{3}=0
$$

where

$$
\begin{gathered}
a_{1}=m+\frac{\beta_{p} S^{*} Y^{*}}{I^{*}}+\varphi^{\prime}\left(I^{*}\right) I^{*}-\mu K-d I^{*}, \\
a_{2}=\left(\beta_{p} Y^{*}+\beta_{s} I^{*}\right)\left(\beta_{s} S^{*} K-d\right)-\beta_{p} S^{*}\left(\frac{\Lambda \beta_{1}}{m}-\beta_{1} Y^{*}\right) \\
-\left(\mu K+d I^{*}\right)\left(\frac{\beta_{p} S^{*} Y^{*}}{I^{*}}+\varphi^{\prime}\left(I^{*}\right) I^{*}\right)-m\left(\mu K+d I^{*}\right), \\
a_{3}=m\left(\mu K+d I^{*}\right)\left(-\frac{\beta_{p} S^{*} Y^{*}}{I^{*}}-\varphi^{\prime}\left(I^{*}\right) I^{*}\right)-\beta_{p} S^{*}\left(\beta_{p} Y^{*}+\beta_{s} I^{*}\right)\left(\frac{\Lambda \beta_{1}}{m}-\beta_{1} Y^{*}\right) \\
-m\left(\beta_{p} Y^{*}+\beta_{s} I^{*}\right)\left(-\left(\beta_{s} S^{*} K-d\right)\right)+\beta_{p} S^{*}\left(\frac{\Lambda \beta_{1}}{m}-\beta_{1} Y^{*}\right)\left(\mu K+d I^{*}\right) .
\end{gathered}
$$

Since $\varphi$ is nondecreasing, $\varphi^{\prime}\left(I^{*}\right) \geq 0$. This implies $a_{1}>0, a_{2}>0, a_{3}>0$.

Then

$$
\begin{aligned}
a_{1} a_{2}-a_{3} & {\left[m+\frac{\beta_{p} S^{*} Y^{*}}{I^{*}}+\varphi^{\prime}\left(I^{*}\right) I^{*}-\mu K-d I^{*}\right]\left[\left(\beta_{p} Y^{*}+\beta_{s} I^{*}\right)\left(\beta_{s} S^{*} K-d\right)\right.} \\
& \left.-\beta_{p} S^{*}\left(\frac{\Lambda \beta_{1}}{m}-\beta_{1} Y^{*}\right)-\left(\mu K+d I^{*}\right)\left(\frac{\beta_{p} S^{*} Y^{*}}{I^{*}}+\varphi^{\prime}\left(I^{*}\right) I^{*}\right)-m\left(\mu K+d I^{*}\right)\right] \\
& -\left[m\left(\mu K+d I^{*}\right)\left(-\frac{\beta_{p} S^{*} Y^{*}}{I^{*}}-\varphi^{\prime}\left(I^{*}\right) I^{*}\right)-\beta_{p} S^{*}\left(\beta_{p} Y^{*}+\beta_{s} I^{*}\right)\left(\frac{\Lambda \beta_{1}}{m}-\beta_{1} Y^{*}\right)\right. \\
& \left.-m\left(\beta_{p} Y^{*}+\beta_{s} I^{*}\right)\left(-\left(\beta_{s} S^{*} K-d\right)\right)+\beta_{p} S^{*}\left(\frac{\Lambda \beta_{1}}{m}-\beta_{1} Y^{*}\right)\left(\mu K+d I^{*}\right)\right] \\
> & \left(\mu K+d I^{*}\right)\left[\left(\mu K+d I^{*}\right)\left(\frac{\beta_{p} S^{*} Y^{*}}{I^{*}}+\varphi^{\prime}\left(I^{*}\right) I^{*}\right)+m\left(\mu K+d I^{*}\right)\right] \\
& -m\left(\beta_{p} Y^{*}+\beta_{s} I^{*}\right)\left(\beta_{s} S^{*} K-d\right)>0
\end{aligned}
$$

Hence, all of the Routh-Hurwitz criteria are satisfied. Thus it follows that the endemic equilibrium $P^{*}$ of (2.3), which exists if $R_{0}>1$, is always locally asymptotically stable. The proof is completed. $\square$

Next, we will prove global stability of the disease-free equilibrium and endemic equilibrium of (2.3). We need to use the LaSalle-type invariance principle for the differential inclusion (Theorem 3 in [19]) to prove their global stability.

Let $x=S-K$. We obtain the following system analogous to (2.5)

$$
\left\{\begin{array}{l}
\dot{x}=-\left(\mu+\beta_{p} Y+\beta_{s} I\right) x-\left(\beta_{s} K-d\right) I-\beta_{p} K Y, \\
\dot{I} \in\left(\beta_{p} Y+\beta_{s} I\right) x+\left(\beta_{s} K-\omega\right) I+\beta_{p} K Y-\overline{c o}[h(I)], \\
\dot{Y}=\frac{\Lambda \beta_{1} I}{m}-\left(\beta_{1} I+m\right) Y .
\end{array}\right.
$$


Set

$$
V_{1}(x, I, Y)=x+I+\frac{\beta_{p} K}{m} Y
$$

and

$$
\boldsymbol{G}(x, I, Y)=\left(\begin{array}{c}
-\left(\mu+\beta_{p} Y+\beta_{s} I\right) x-\left(\beta_{s} K-d\right) I-\beta_{p} K Y \\
\left(\beta_{P} Y+\beta_{s} I\right) x+\left(\beta_{s} K-\omega\right) I+\beta_{p} K Y-\overline{c o}[h(I)] \\
\frac{\Lambda \beta_{1} I}{m}-\left(\beta_{1} I+m\right) Y
\end{array}\right)
$$

For any $\boldsymbol{v}=\left(v_{1}, v_{2}, v_{3}\right)^{\mathrm{T}} \in G(x, I, Y)$, there exists an $\eta(t) \in \overline{c o}[h(I)]$ such that

$$
\boldsymbol{v}=\left(\begin{array}{c}
-\left(\mu+\beta_{p} Y+\beta_{s} I\right) x-\left(\beta_{s} K-d\right) I-\beta_{p} K Y \\
\left(\beta_{p} Y+\beta_{s} I\right) x+\left(\beta_{s} K-\omega\right) I+\beta_{p} K Y-\eta(I) I \\
\frac{\Lambda \beta_{1} I}{m}-\left(\beta_{1} I+m\right) Y
\end{array}\right)
$$

Hence,

$$
\begin{aligned}
& \nabla V_{1}(x, I, Y) \cdot \boldsymbol{v} \\
& =\left(1,1, \frac{\beta_{p} K}{m}\right)\left(\begin{array}{c}
-\left(\mu+\beta_{p} Y+\beta_{s} I\right) x-\left(\beta_{s} K-d\right) I-\beta_{p} K Y \\
\left(\beta_{p} Y+\beta_{s} I\right) x+\left(\beta_{s} K-\omega\right) I+\beta_{p} K Y-\eta(I) I \\
\frac{\Lambda \beta_{1} I}{m}-\left(\beta_{1} I+m\right) Y
\end{array}\right) \\
& =-\mu x+d I-\left(\omega+\eta(I)-\frac{K \Lambda \beta_{p} \beta_{1}}{m^{2}}\right) I-\frac{K \Lambda \beta_{p} \beta_{1} I Y}{m} \\
& <-\mu x-\left(\omega+\eta(I)-\frac{K \Lambda \beta_{p} \beta_{1}}{m^{2}}\right) I-\frac{K \Lambda \beta_{p} \beta_{1} I Y}{m}
\end{aligned}
$$

When $R_{0} \leq 1$, the nondecreasing of $\varphi$ implies

$$
\omega+\varphi(0)-\frac{K \Lambda \beta_{p} \beta_{1}}{m^{2}} \geq \omega+\eta(I)-\frac{K \Lambda \beta_{p} \beta_{1}}{m^{2}} \geq 0
$$

It shows that $V_{1}$ is a Lyapunov function of (4.23).

Furthermore, when $R_{0}<1$, we have

$$
\begin{aligned}
\mathcal{Z}_{V_{1}} & =\left\{(x, I, Y) \in \mathbb{R}^{3}: \nabla V_{1}(x, I, Y) \cdot \boldsymbol{v}=0, \boldsymbol{v} \in \boldsymbol{G}(x, I, Y)\right\} \\
& =\{(0,0, Y): Y \geq 0\}
\end{aligned}
$$

When $I=0$, we have $\frac{\mathrm{d} Y}{\mathrm{~d} t}=-m Y$, which implies $\lim _{t \rightarrow \infty} Y(t)=0$. For any $l>0$, we set

$$
V_{1}^{l} \triangleq\left\{(x, I, Y) \in \mathbb{R}^{3}: \nabla V_{1}(x, I, Y) \leq l\right\}
$$

Hence, the largest weakly invariant subset of $\overline{\mathcal{Z}_{V_{1}}} \cap V_{1}^{l}$ is the singleton 
$M=\{(0,0,0)\}$.

When $I=0$, we have

$$
\mathcal{Z}_{V_{1}}=\{(0,0, Y): Y \geq 0\} \bigcup\{(0, I, Y): \eta(t)=\varphi(0), I>0\}
$$

From the first equation of (4.23) and $\mathrm{x}=0$, it may be concluded that $I=0$. Therefore, we see that the largest weakly invariant subset of $\overline{\mathcal{Z}_{V_{1}}} \cap V_{1}^{l}$ is also the singleton $M=\{(0,0,0)\}$. By the LaSalle-type invariance principle, the equilibrium $(0,0,0)$ of (4.23) is globally asymptotically stable as $R_{0} \leq 1$. Summarizing the above analysis, we obtain the following theorem.

Next, we demonstrate the global stability of the endemic equilibrium $P^{*}$ of (2.3). So, we have the following theorem.

Theorem 4.4. Suppose that assumption (H1) holds. If $R_{0} \leq 1$, the disease-free equilibrium $P_{0}$ of the system (2.3) is globally asymptotically stable.

Proof: Let

$$
V(S, I, Y)=\left(S-S^{*}-S \ln \frac{S}{S^{*}}\right)+\left(I-I^{*}-I^{*} \ln \frac{I}{I^{*}}\right)+\left(Y-Y^{*}-Y^{*} \ln \frac{Y}{Y^{*}}\right)
$$

Write

$$
\eta^{*}=(\omega-d) \in \overline{c o}\left[\varphi\left(I^{*}\right)\right]
$$

and

$$
\boldsymbol{H}(S, I, Y)=\left(\begin{array}{c}
\mu(K-S)-\left(\beta_{P} Y+\beta_{s} I\right) S+d I \\
\left(\beta_{P} Y+\beta_{s} I\right) S-\omega I-\overline{c o}[h(I)] \\
\frac{\Lambda \beta_{1} I}{m}-\left(\beta_{1} I+m\right) Y
\end{array}\right)
$$

For any $\boldsymbol{v}=\left(v_{1}, v_{2}, v_{3}\right)^{\mathrm{T}} \in \boldsymbol{H}(S, I, Y)$, there exists an $\eta(t) \in \overline{c o}[h(I)]$ such that

$$
\boldsymbol{v}=\left(\begin{array}{c}
\mu(K-S)-\left(\beta_{P} Y+\beta_{s} I\right) S+d I \\
\left(\beta_{P} Y+\beta_{s} I\right) S-\omega I-\eta(I) I \\
\frac{\Lambda \beta_{1} I}{m}-\left(\beta_{1} I+m\right) Y
\end{array}\right)
$$

Hence

$$
\begin{aligned}
& \nabla V(S, I, Y) \cdot \boldsymbol{v}=\left(1,1,1-\frac{Y^{*}}{Y}\right)\left(\begin{array}{c}
\mu(K-S)-\left(\beta_{P} Y+\beta_{s} I\right) S+d I \\
\left(\beta_{P} Y+\beta_{s} I\right) S-\omega I-\eta(I) I \\
\frac{\Lambda \beta_{1} I}{m}-\left(\beta_{1} I+m\right) Y
\end{array}\right) \\
& =\mu(K-S)+d I-\omega I-\eta(I) I+\frac{\Lambda \beta_{1} I}{m}-\left(\beta_{1} I+m\right) Y-\frac{\Lambda \beta_{1} I Y^{*}}{m Y}+\left(\beta_{1} I+m\right) Y^{*} \\
& =\left(\eta^{*}-\eta(I)\right) I+\frac{\Lambda \beta_{1} I}{m}\left(1-\frac{Y^{*}}{Y}\right)+\left(\beta_{1} I+m\right)\left(Y^{*}-Y\right)
\end{aligned}
$$


The monotonicity of $\varphi$ implies $\eta^{*}-\eta(I) \leq 0$. Thus $\nabla V(S, I, Y) \cdot v \leq 0$. This shows that $\mathrm{V}$ is a Lyapunov function of (2.24). Define

$$
\begin{aligned}
\mathcal{Z}_{V} & =(S, I, Y) \in \mathbb{R}^{3}: \nabla V_{1}(S, I, Y) \cdot \boldsymbol{v}=0, \boldsymbol{v} \in \boldsymbol{H}(S, I, Y) \\
& =\left\{\left(S^{*}, I^{*}, Y^{*}\right)\right\} \cup\left\{\left(S^{*}, I, Y\right): \frac{I}{I^{*}}=\frac{Y}{Y^{*}}, \eta^{*}=\eta(t)\right\}
\end{aligned}
$$

If $S=S^{*}$, then the first equation of (2.6) implies $I=I^{*}, Y=Y^{*}$. Consequently, for any $l>0$, the largest weakly invariant subset of $\overline{\mathcal{Z}_{V}} \cap V^{l}$ of (2.6) is the singleton overline $\left\{\left(S^{*}, I^{*}, Y^{*}\right)\right\}$. Here

$$
V^{l}=\left\{\left(S-S^{*}, I-I^{*}, Y-Y^{*}\right) \in \mathbb{R}^{3}: V(S, I, Y) \leq l\right\}
$$

Therefore, $P^{*}$ is globally asymptotically stable if $R_{0}>1$. This completes the proof. $\square$

\section{Numerical Simulation}

To make our analysis more intuitive, some numerical simulations of solutions of the model (2.6) is provided which to illustrate the influence of insect vector and discontinuous treatment on the spread of plant disease. We apparent a treatment function satisfying $\left(\mathrm{H}_{1}\right)$ as follows:

$$
h(I)=\left\{\begin{array}{l}
c_{1} I, I \leq I_{0} \\
c_{2} I, I>I_{0}
\end{array}\right.
$$

where $0 \leq c_{1}<c_{2}$. The treatment function $h(I)$ is applied at the following case: when the infective individuals $I$ attain some threshold $I_{0}$, the treatment rate should be strengthened to control the spread of the plant disease.

To better illustrate the effects of non-continuous healing on the spread of plant disease, the following parameters are derived from [16]. Let $\beta_{1}=0.0025$, $\beta_{p}=0.0025, \quad \beta_{s}=0.0001, \mu=0.1, \gamma=0.4, K=1000, \quad c_{1}=0, \quad \Lambda=5$, $m=0.3, d=0.1$, then we easily calculate $R_{0}=0.3578<1$ by using (4.5). Figure 1 shows that the infective individuals $I$ and the infective insect vectors $Y$ tend to 0 , and it means that the disease goes to extinction. In addition, we find that the peak values of the infective is affected by the different values of $c_{2}$. Figure 1 shows that larger values of $c_{2}$ can reduce the peak values of the infective. Therefore, we can increase the treatment rate to prevent the spread of disease after the number infective individuals reaching some high level. From Figure 1, the infective individuals reach some level and strengthening the treatment rate is also effective for disease control, even though we do not take any treatment measures at the initial time of the diseases outbreak.

If we fixed all parameter values as follows: $\beta_{1}=0.01, \beta_{p}=0.02, \beta_{s}=0.01$, $\mu=0.1, \gamma=0.4, K=1000, \Lambda=5, m=0.3, d=0.1$ one could easily see that $R_{0}=16.8889>1$, by using (4.5). Figure 2 shows that the endemic equilibrium is globally asymptotically stable. By (4.5), it's obvious find that the basic reproductive number $R_{0}$ is independent of $c_{2}$, and the different values of $c_{2}$ can affect the stability level of the infective. That is to say, larger values of 


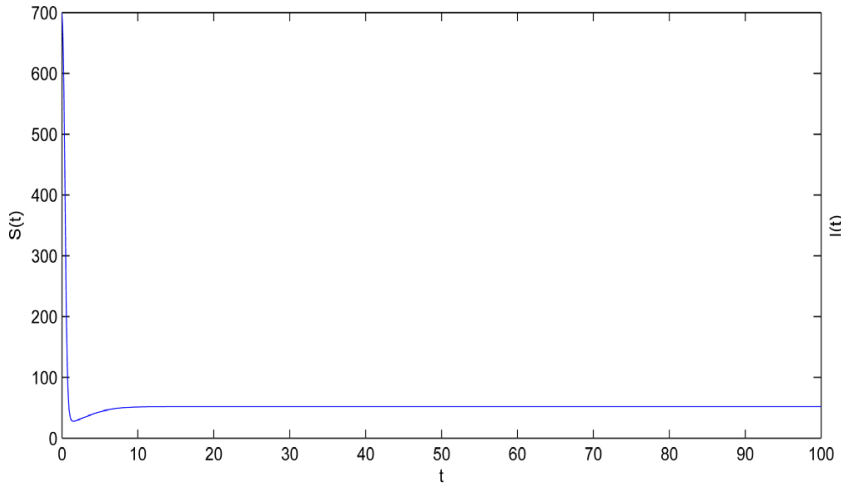

(a)

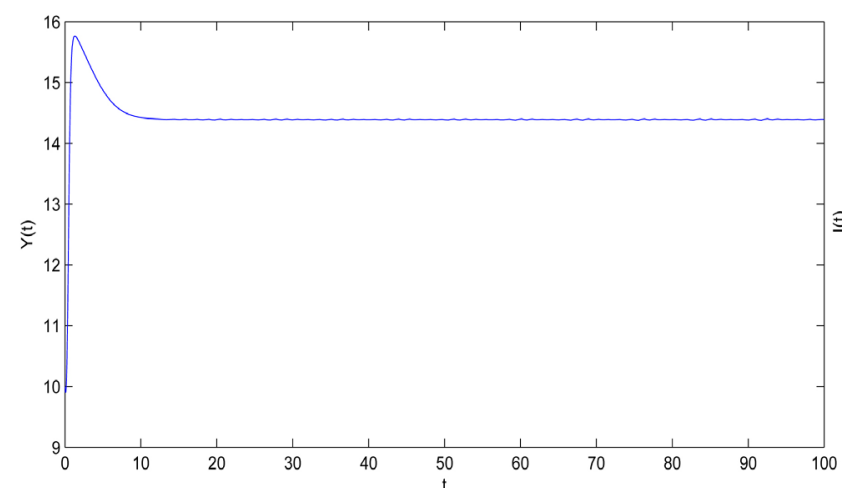

(c)

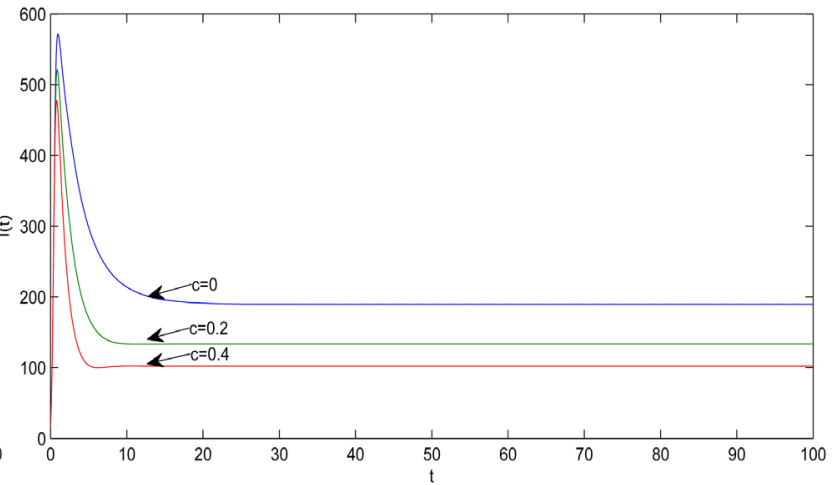

(b)

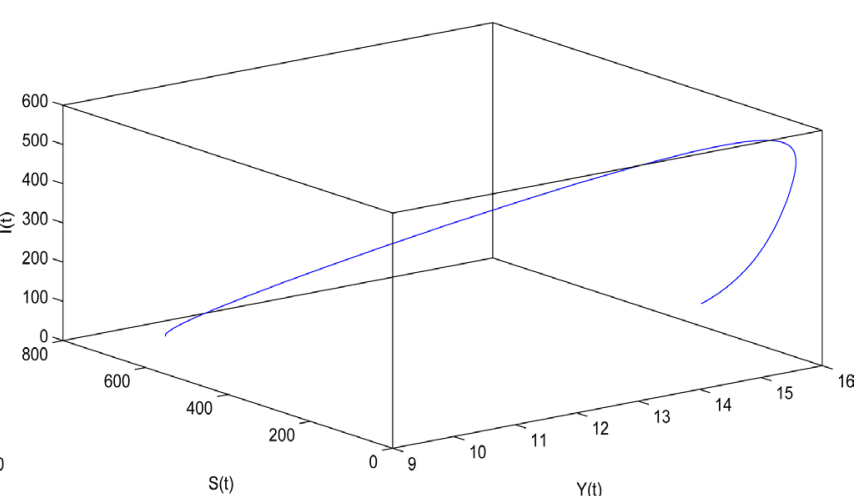

(d)

Figure 1. Stability of disease-free equilibrium. The parameters are fixed as follows: $\beta_{1}=0.0025, \beta_{p}=0.0025, \beta_{s}=0.0001$, $\mu=0.1, \gamma=0.4, K=1000, c_{1}=0, \Lambda=5, m=0.3, d=0.1$, and the initial values are $\left(S_{0}, I_{0}, Y_{0}\right)=(700,15,10)$. The time series charts for $S(t), I(t), Y(t)$ and the phase diagram are given in (a), (b), (c), and (d), respectively.

$c_{2}$ can impact level of the infective. It implies that the strengthening of the treatment rate can effectively control the spread of plant disease after the number of infective individuals has increased to some high level.

\section{Discussion}

As for the plant infectious disease model, our main object is to investigate the effect of the insect vector and discontinuous treatment function on the dynamics of spreading the plant disease. We calculated the basic reproduction number $R_{0}$, which is derived under some reasonable assumptions on the discontinuous treatment function. It is an important threshold parameter which plays an important role in determining the global dynamics of the model (2.6) and whether it persists or dies out of the disease. When $R_{0} \leq 1$, the disease-free equilibrium is globally stable, which means that the disease always dies out, and when $R_{0}>1$, the plant disease will be permanent which means that after some period of time the plant disease will become endemic and it is global stable.

In this paper, we studied the existence, local stability and global stability of the disease-free equilibrium and endemic equilibrium of the system (2.3) in detail. By building a suitable Lyapunov function, and the Jacobian matrix method, 


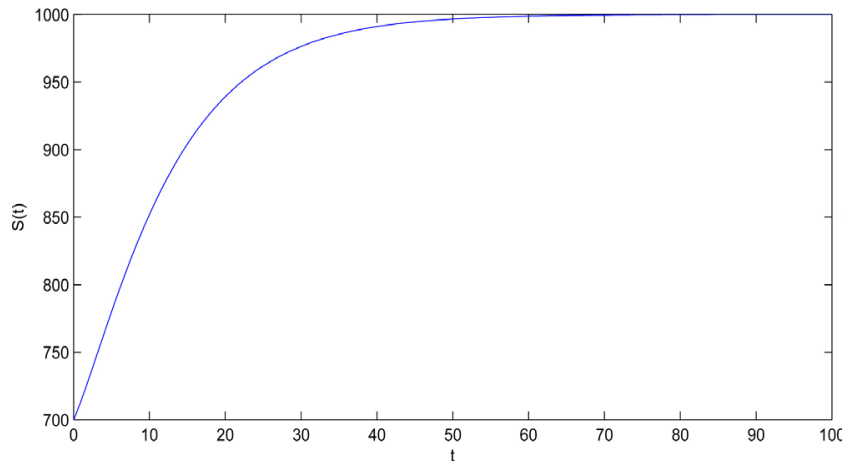

(a)

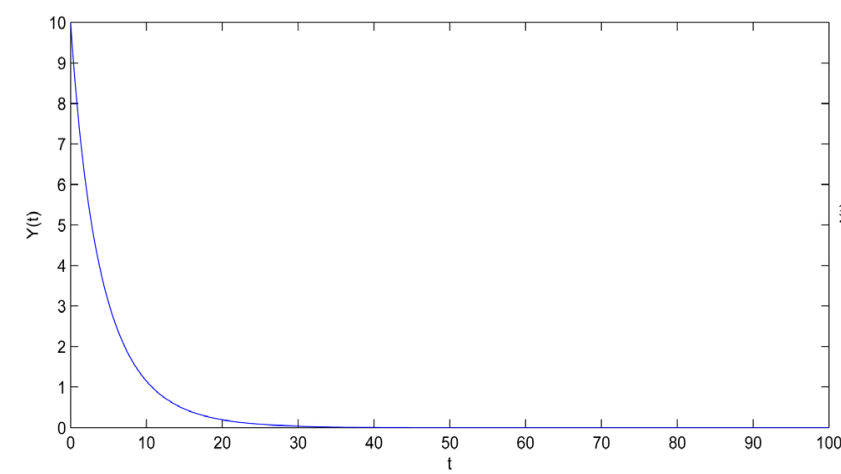

(c)

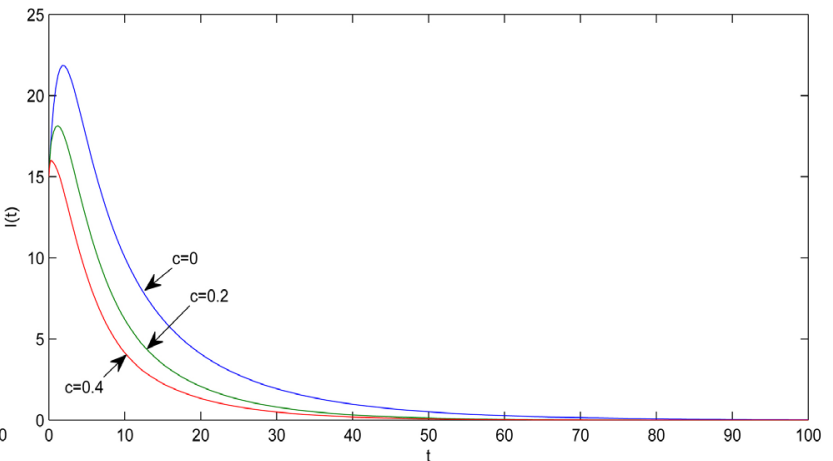

(b)

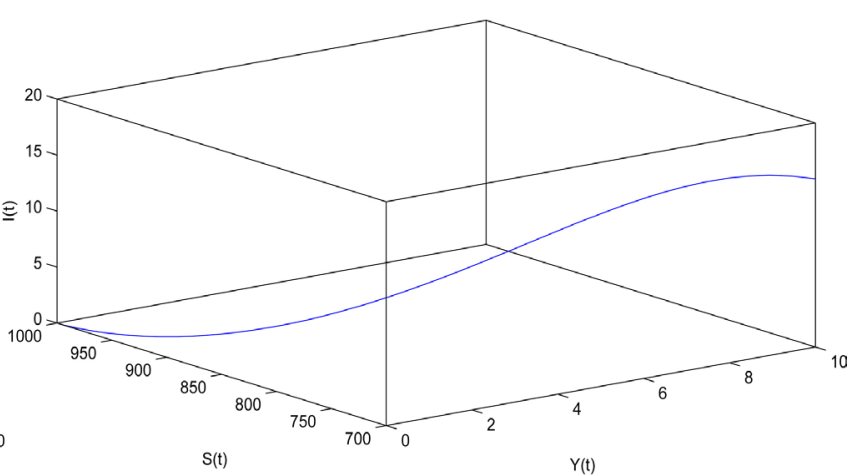

(d)

Figure 2. Stability of endemic equilibrium. The parameters are fixed as follows: $\beta_{1}=0.01, \beta_{p}=0.02, \quad \beta_{s}=0.01, \mu=0.1$, $\gamma=0.4, K=1000, \Lambda=5, m=0.3, d=0.1$. The time series charts for $\mathrm{S}(\mathrm{t}), \mathrm{I}(\mathrm{t}), \mathrm{Y}(\mathrm{t})$ and the phase diagram are given in $(\mathrm{a})$, (b), (c), and (d), respectively.

employing Routh-Hurwitz criteria and LaSalle-type invariance principle, the main results as shown in Theorems 4.2, 4.3 and 4.4 have been derived. Our main results indicate that if $R_{0}<1$, then the disease-free equilibrium is globally asymptotically stable, if $R_{0}>1$, the unique endemic equilibrium is globally asymptotically stable of the system (2.3). From above results, it is easy to find that the basic reproduction number $R_{0}$ plays an important role in determining the persistence or dying out of the disease.

\section{Acknowledgements}

The research have been supported by The Natural Science Foundation of China (11561004), the Science and Technology research project of Jiangxi Provincial Education Department (171373, 171374, GJJ170815), The bidding project of Gannan Normal University (16zb02).

\section{References}

[1] Lee, J.A., Halbert, S.E., Dawson, W.O., et al. (2015) Asymptomatic Spread of Huanglongbing and Implications for Disease Control. Proceedings of the National Academy of Sciences, 112, 7605-7610. https://doi.org/10.1073/pnas.1508253112

[2] Smith, A.B., Beeck, C.P., Cowling, W.A., et al. (2013) A Bivariate Mixed Model Approach for the Analysis of Plant Survival Data. Euphytica, 190, 371-383. 
https://doi.org/10.1007/s10681-012-0791-0

[3] Xia, L., Gao, S., Zou, Q., et al. (2013) Analysis of a Nonautonomous Plant Disease Model with Latent Period. Applied Mathematics and Computation, 223, 147-159. https://doi.org/10.1016/j.amc.2013.08.011

[4] Wang, J., Zhang, F., Wang, L., et al. (2016) Equilibrium, Pseudoequilibrium and Sliding-Mode Heteroclinic Orbit in a Filippov-Type Plant Disease Model. Nonlinear Analysis: Real World Applications, 31, 308-324. https://doi.org/10.1016/j.nonrwa.2016.01.017

[5] Zhao, W., Li, J., Zhang, T., et al. (2017) Persistence and Ergodicity of Plant Disease Model with Markov Conversion and Impulsive Toxicant Input. Communications in Nonlinear Science and Numerical Simulation, 48, 70-84. https://doi.org/10.1016/j.cnsns.2016.12.020

[6] Jackson, M. and Chen-Charpentier, B.M. (2016) Modeling Plant Virus Propagation with Delays. Journal of Computational and Applied Mathematics, 2016, 1-14.

[7] Xue, Y. and Wang, J. (2012) Backward Bifurcation of an Epidemic Model with Infectious Force in Infected and Immune Period and Treatment. Abstract and Applied Analysis, 2012, Article ID: 647853. https://doi.org/10.1155/2012/647853

[8] Hussaini, N. and Winter, M. (2010) Travelling Waves for an Epidemic Model with Non-Smooth Treatment Rates. Journal of Statistical Mechanics, 11, Article ID: 11019. https://doi.org/10.1088/1742-5468/2010/11/P11019

[9] Brauer, F. (2008) Epidemic Models with Heterogeneous Mixing and Treatment. Bulletin of Mathematical Biology, 70, 1869-1885. https://doi.org/10.1007/s11538-008-9326-1

[10] Hu, Z., Liu, S. and Wang, H. (2008) Backward Bifurcation of an Epidemic Model with Standard Incidence Rate and Treatment Rate. Nonlinear Analysis. Real World Applications, 9, 2302-2312. https://doi.org/10.1016/j.nonrwa.2007.08.009

[11] Wang, W. and Ruan, S. (2004) Bifurcations in an Epidemic Model with Constant Removal Rate of the Infectives. Journal of Mathematical Analysis and Applications, 291, 775-793. https://doi.org/10.1016/j.jmaa.2003.11.043

[12] Wang, W. (2006) Backward Bifurcation of an Epidemic Model with Treatment. Mathematical Biosciences, 201, 58-71. https://doi.org/10.1016/j.mbs.2005.12.022

[13] Guo, Z. Huang, L. and Zou, X. (2012) Impact of Discontinuous Treatments on Disease Dynamics in an SIR Epidemic Model. Mathematical Biosciences and Engineering, 9, 97-110. https://doi.org/10.3934/mbe.2012.9.97

[14] Zhang, T., Kang, R., Wang, K. and Liu, J. (2015) Global Dynamics of an SEIR Epidemic Model with Discontinuous Treatment. Advances in Difference Equations, 361, 1-16.

[15] Huang, L., Guo, Z. and Wang, J. (2011) Theory and Applications of Differential Equations with Discontinuous Righthand Sides. Science Press, Beijing. (In Chinese)

[16] Shi, R., Zhao, H. and Tang, S. (2014) Global Dynamic Analysis of a Vector-Borne Plant Disease Model. Advances in Difference Equations, 59, 1-16. https://doi.org/10.1186/1687-1847-2014-59

[17] Filippov, A.F. (1988) Differential Equations with Discontinuous Righthand Sides. Mathematics and Its Applications (Soviet Series). Kluwer Academic, Boston. https://doi.org/10.1007/978-94-015-7793-9

[18] Ma, Z. and Zhou, C. (2016) Methods of Qualitative and Stability of Ordinary Differential Equations. Science Press, Beijing. (In Chinese)

[19] Baciotti, A. and Ceragioli, F. (1999) Stability and Stabilization of Discontinuous Systems and Non-Smooth Lyapunov Function. ESAIM: Control, Optimisation and Calculus of Variations, 4, 361-376. https://doi.org/10.1051/cocv:1999113 